\title{
DESENVOLVIMENTO HUMANO LOCAL: UMA ANÁLISE MULTIVARIADA PARA OS PEQUENOS MUNICÍPIOS EM MINAS GERAIS
}

\author{
LOCAL HUMAN DEVELOPMENT: \\ A MULTIVARIATE ANALYSIS OF SMALL MUNICIPALITIES IN MINAS GERAIS \\ DESARROLLO HUMANO LOCAL: \\ UN ANÁLISIS MULTIVARIADO PARA LOS PEQUEÑOS MUNICIPIOS DE MINAS GERAIS
}

\begin{abstract}
Resumo
Este trabalho busca explorar de forma comparativa a realidade socioeconômica dos pequenos municípios de Minas Gerais, sob a perspectiva do desenvolvimento humano local e com base nas informações e nos dados disponíveis dos anos 2000 a 2010 . Para atingir os objetivos propostos, utilizaram-se as técnicas de análise de cluster e de regressão por Mínimos Quadrados Ordinários. A classificação dos municípios com base na análise de cluster revelou três agrupamentos, que foram caracterizados como desenvolvimento humano local baixo, intermediário e alto. Os resultados apontam para a relativa importância da condição econômica, do nível de concentração da renda e da capacidade fiscal do município quanto à taxa de crescimento do desenvolvimento humano dos pequenos municípios. A partir do exposto, reforça-se a necessidade de se buscar um desenvolvimento equilibrado por meio do fortalecimento das ligações entre crescimento econômico e desenvolvimento humano.
\end{abstract}

Palavras-chaves: Desenvolvimento humano local, Minas Gerais, pequenos municípios, Índice de Desenvolvimento Humano Municipal, análise de cluster.

Valéria de Jesus Coelho Ferreira dos Santos - valcoelh02003@yahoo.com.br

Mestre em Administração Pública pela Fundação João Pinheiro, Escola de Governo - Belo Horizonte - MG, Brasil

Frederico Poley Martins Ferreira - frederico.poley@fjp.mg.gov.br

Professor da Fundação João Pinheiro, Escola de Governo - Belo Horizonte - MG, Brasil

Submetido 06.04.2016. Aprovado 13.01.2017

DOI: http://dx.doi.org/10.12660/cgpc.v22n71.60417

Esta obra está submetida a uma licença Creative Commons 


\section{Abstract}

This paper aims to provide a comparative analysis of the socioeconomic reality of small municipalities in the Brazilian state of Minas Gerais from the perspective of municipal human development, based on data from 2000 and 2010. To that end, we used cluster analysis and regression by OLS. The classification of municipalities in the cluster analysis revealed three clusters or groups, namely, low, intermediate and high local human development. Results indicate a relative importance of local economic conditions, as well as municipalities' income concentration level and fiscal capacity, for increasing human development rate. Therefore, the study reinforces the importance of seeking balanced development by strengthening the links between economic growth and human development.

Keywords: Human development, Minas Gerais, municipalities, Municipal Human Development Index cluster.

\section{Resumen}

Este estudio tiene como objetivo explorar comparativamente la realidad socioeconómica de los pequeños municipios de Minas Gerais desde la perspectiva del desarrollo humano local, basado en información de 2000 y 2010. Para lograr los objetivos propuestos, se utilizaron las técnicas analíticas clúster y la regresión por MCO. La clasificación de los municipios con base en el análisis de conglomerados revelo tres agrupamientos que se caracterizaron como desarrollo humano local bajo, intermedio y alto. Los resultados apuntan una relativa importancia de las condiciones económicas, del nivel de concentración de ingresos y de la capacidad fiscal del municipio respecto a la tasa de crecimiento del desarrollo humano de los pequeños municipios. De lo anterior, se refuerza la necesidad de buscar un desarrollo equilibrado mediante el fortalecimiento de los vínculos entre crecimiento económico y desarrollo humano.

Palabras clave: Desarrollo Humano, Minas Gerais, pequeños municipios, Índice de Desarrollo Humano Municipal, análisis de clúster.

\section{INTRODUÇÃO}

A discussão sobre o tema desenvolvimento não é trivial, dada a busca pelo desenvolvimento social e econômico como objeto de aspiração de países e regiões. Por meio do primeiro Relatório de Desenvolvimento Humano Global, em 1990, o Programa das Nações Unidas para o Desenvolvimento (PNUD) propôs a abordagem do desenvolvimento humano, cujo objetivo era melhorar a vida humana.

Nessa perspectiva, a análise do bem-estar do indivíduo se tornou uma medida comparável entre os países com a criação do Índice de Desenvolvimento Humano (IDH). Também foi criado o Índice de Desenvolvimento Humano Municipal (IDHM) para a compreensão da realidade dos municípios.

A proposta do IDHM retrata o reconhecimento da heterogeneidade do país e a ne- cessidade de fazer a distinção das especificidades locais para subsidiar a formulação de políticas públicas. Para os aspectos deste estudo, definiu-se como critério de local o município, menor unidade político administrativa do país.

Diante do exposto, desperta considerável interesse a situação dos municípios de pequeno porte, em razão de fatores específicos relacionados às suas potencialidades e limitações, os quais são apresentados na literatura. Nesta pesquisa, o porte do município foi definido a partir do tamanho de sua população. Considera-se pequeno aquele que apresentou população igual ou inferior a 50.000 habitantes em 2010. É interessante observar que a importância desse grupo se amplia quando se observa sua representatividade no total de municípios brasileiros, $89 \%$, em 2010. No caso de Minas Gerais, dos 853 municípios, 787 têm até 50.000 habitantes, ou seja, $92 \%$. 
Nesse contexto, procura-se explorar de forma comparativa a realidade socioeconômica dos pequenos municípios mineiros sob a perspectiva do desenvolvimento humano local. A proposta deste estudo justifica-se por sua importância para a área de Administração Pública, especialmente, pela possibilidade de subsidiar os formuladores de políticas públicas no direcionamento de novas propostas de atuação a favor de um projeto de desenvolvimento local capaz de dinamizar a economia e promover ganhos em termos de desenvolvimento humano.

O trabalho está organizado em quatro seções além desta introdução. Na primeira, apresenta-se a fundamentação teórica, destacando-se a emergência da abordagem do desenvolvimento humano, a proposta de Desenvolvimento Humano Local e as especificidades dos pequenos municípios. Em seguida, expõe-se a metodologia, a análise dos resultados obtidos por meio do método cluster e de regressão e por fim, expõem-se as considerações finais.

\section{FUNDAMENTAÇÃO TEÓRICA}

\section{Desenvolvimento humano}

A publicação do Relatório do Desenvolvimento Humano pelo Programa das Nações Unidas para o Desenvolvimento (PNUD), em 1990, foi um ponto de inflexão na discussão teórica sobre o desenvolvimento. $O$ PNUD conceituou desenvolvimento humano como o "processo de ampliação das escoIhas e liberdades das pessoas para que elas tenham capacidades e oportunidades para ser aquilo que desejam ser" (PNUD \& FJP \& IPEA, 2013, p. 1).
Esse conceito qualifica como objetivo do desenvolvimento a melhoria das vidas humanas por meio da expansão das possibilidades de ser e de fazer do indivíduo. Para alcançar essa meta, é preciso remover obstáculos como analfabetismo, falta de saúde, impossibilidade de acesso a recursos e ausência de liberdades civis e políticas (Fukuda-Parr, 2002).

O crescimento econômico também se apresenta como estratégia necessária para gerar os recursos essenciais para promoção do desenvolvimento humano. Esses recursos são importantes, pois se busca um crescimento que propicie escolhas mais completas para todos em detrimento da opção de apenas algumas para a maioria ou muitas delas para alguns. No entanto, a ligação entre crescimento e desenvolvimento não é direta, o que torna essencial dar atenção à estrutura e à qualidade do crescimento (PNUD, 1996).

Busca-se, assim, um tipo de crescimento que inclua as pessoas no processo de evolução, que seja capaz de gerar pleno emprego e segurança dos meios de subsistência, que estimule a liberdade e o empoderamento das pessoas, que distribua benefícios equitativamente, que colabore para coesão social e a cooperação e que garanta o desenvolvimento humano futuro. Em contraponto, deve-se evitar o crescimento sem criação de empregos, desumano, sem direito de opinião, desenraizado e sem futuro.

Cardoso (1996) argumenta que o estabelecimento de um padrão de crescimento que promova desenvolvimento humano nem sempre é uma alternativa clara. Acrescenta 
ainda o desafio de o Estado responder às maiores demandas por políticas sociais em um contexto de reformulação de seu papel.

Segundo PNUD (1996), para um desenvolvimento equilibrado, as ligações entre crescimento e desenvolvimento humano precisam ser fortalecidas. Os pontos significativos que determinam como o crescimento pode contribuir para o desenvolvimento são: equidade, oportunidade de emprego, acesso aos ativos produtivos, igualdade entre os sexos, política populacional, bom governo, uma sociedade civil ativa.

\section{Desenvolvimento humano local}

Pensar no desenvolvimento humano em âmbito local é reconhecer as singularidades de cada lugar, principalmente no Brasil, que apresenta grande extensão territorial e diversidade social, cultural e econômica. Também é reconhecer as peculiaridades impostas por um sistema político-administrativo descentralizado, que confira a cada local autonomia de ações em favor do bem-estar da sua população. Assinala-se que esse reconhecimento não implica desconsiderar o contexto geral dessa população.

Buarque (1999, p. 26) lembra que "o local e o municipal são subsistemas de um sistema mais amplo e global, de tal modo que os problemas e as potencialidades municipais e locais dependem de fatores externos e das condições do contexto". Por esse motivo, as decisões em nível nacional são importantes, dada as inúmeras possibilidades que estão ao alcance da União para intervenção na sociedade e na economia, como políticas monetárias e fiscais, questões institucionais (leis trabalhistas, direito de proprie- dade e contrato etc.), sistema de educação, políticas sociais e outras. O contexto global, por sua vez, marcado pela interligação entre os mercados, conduz à interdependência econômica entre local e global.

Na concepção de Boisier (2001), desenvolvimento local alude a uma modalidade de desenvolvimento que pode tomar forma em determinado território. Na maioria dos estudos, por simplificação, como é o caso deste estudo, relaciona-se o adjetivo local à ideia de município, independentemente do seu tamanho. $\mathrm{O}$ autor adverte que a referência de local só faz sentido a partir do ponto de observação. Assim, as regiões constituem espaços locais de países; os estados formam locais de regiões, e os municípios compõem locais de estados.

Tanto no Brasil quanto em outros países da América Latina, o movimento de descentralização político-administrativa foi incentivado. Acreditava-se que a descentralização da gestão pública seria a melhor alternativa para ampliar a eficiência do uso dos recursos, a eficácia das políticas públicas, a transparência das decisões e a criação de condições para a responsabilização de seus governantes perante seus cidadãos (Veloso et al., 2011).

No caso brasileiro, a Constituição de 1988 transferiu parte das obrigações sociais para os municípios. Com isso, a administração local tornou-se prestadora, em primeira instância, de serviços de saúde, educação, transporte e saneamento básico (Dowbor, 2008).

Segundo Abrucio e Couto (1996), a capacidade de os municípios responderem aos desafios assumidos na promoção de políticas 
DESENVOLVIMENTO HUMANO LOCAL: UMA ANÁLISE MULTIVARIADA PARA OS PEQUENOS MUNICÍPIOS EM MINAS GERAIS

de bem-estar social e promoverem o crescimento econômico com base em ações de âmbito local está relacionada, entre outros fatores, às diferentes características socioeconômicas de cada ente local e à estrutura fiscal da federação brasileira.

No processo de descentralização, os municípios não partiram de um mesmo estágio socioeconômico para assumir os encargos que antes eram de responsabilidade da União ou dos estados. Isso impactou tanto nas diferentes necessidades sociais impostas quanto na capacidade de esses municípios obterem recursos próprios para o financiamento das novas demandas.

No aspecto fiscal, entre as fontes de arrecadação própria (ISS, IPTU, ITBI e taxas), o ISS é um imposto que incide sobre a prestação de serviços. Isso implica que o volume arrecadado é proporcional à base tributária, com estreita relação com o dinamismo da economia local. Dessa forma, os municípios maiores e mais dinâmicos tendem a ter mais recursos próprios advindos da arrecadação do ISS, quando comparados às pequenas cidades e às mais pobres (Abrucio \& Couto, 1996). A dificuldade de geração de recursos próprios eleva para muitos municípios a dependência ao Fundo de Participação dos Municípios (FPM).

\section{Caso especial: os pequenos municípios}

Conforme Ferreira (1996), a importância dos municípios de pequeno porte tem se consolidado nos últimos anos em decorrência de vários fatores. O primeiro deles é a sua relevância em número. Com base no censo de 2010, esse grupo corresponde a $89 \%$ do total de municípios no Brasil, já em Minas
Gerais, alcança $92 \%$ do total de municípios. Os outros fatores estão relacionados à diversidade de contextos, inclusive históricos, à qualidade de vida da população e às oportunidades de desenvolvimento econômico. Também é relevante para esse grupo a questão fiscal e a capacidade de gestão.

O aumento do número de pequenos municípios no fim da década de 1980 e início dos anos 1990 é explicado, em grande parte, pelo processo de ampliação das receitas municipais que acompanhou o processo de descentralização. Após a Constituição Federal (CF) de 1988, foram criados 1.378 municípios, o que representou um aumento de $35 \%$ no total de municípios do país. A maioria deles não tinham 10.000 habitantes (Boueri, Monasterio, Mation, \& Silva, 2013).

O crescimento do número de novos pequenos municípios também foi amplo em Minas Gerais. Evidenciam-se duas mudanças significativas. Primeiro, entre 1960 e 1970, quando houve um incremento de 239 municípios e, depois, entre 1991 e 2000, com a criação de mais 130 municípios. Do total dos novos municípios, $84 \%$ eram pequenos.

Com relação à qualidade de vida e ao desenvolvimento econômico, os avanços dos meios de comunicação e de transporte permitiram a integração dos pequenos municípios ao contexto nacional e internacional sem que eles apresentassem as externalidades negativas inerentes às grandes aglomerações humanas. Em geral, os moradores de pequenas cidades se beneficiam do menor custo de vida em relação ao das maiores cidades. Além disso, os trabalhadores dos pequenos centros contam, pela perspectiva das relações sociais do traba- 
Iho, com vínculos familiares, de amizade e da pequena produção de subsistência, o que não aparece no contexto dos trabalhadores das grandes cidades (Ferreira, 1996).

Apesar do exposto ser uma condição geral, existem diversidades no perfil dos pequenos municípios. Há algumas pequenas cidades com os mesmos problemas dos grandes centros - poluição, desemprego e população marginalizada vivendo em favelas ou em péssimas condições de moradia -, mas também outras com indústrias, boa estrutura de serviços, potencial turístico, presença de universidades e centros de pesquisas (Ferreira, 1996).

A questão fiscal é um ponto relevante para os pequenos municípios. Se, por um lado, alguns foram beneficiados pela maior transferência per capita de recursos por meio do FPM, por outro, enfrentam dificuldades na arrecadação de recursos próprios e maiores gastos per capita para provisão dos serviços básicos em razão da perda de escala.

Souza (2002), ao analisar os resultados dos municípios brasileiros em termos de respostas funcionais e democráticas, encontrou diversidades associadas à heterogeneidade dos municípios em termos de capacidade de corresponder às tarefas que lhes foram impostas. A autora lembra que, em geral, as discussões na literatura tratam os municípios como uniformes e com o mesmo potencial de resposta.

Santos (2013) chama a atenção para o fato de que essa homogeneização no tratamento dos municípios também ocorre no âmbito da legislação brasileira, que não prevê diferenças entre as atribuições dos municípios.
Há poucas exceções, como a obrigatoriedade de apresentar o plano diretor municipal, exigência feita apenas para os municípios com mais de 20.000 habitantes. No caso do estado de Minas Gerais, a situação dos extremos seria semelhante, por exemplo, Belo Horizonte (2,4 milhões de habitantes) e Serra da Saudade (815 habitantes) teriam as mesmas obrigações com a sociedade.

\section{METODOLOGIA}

De acordo com a proposta de classificação apresentada por Gil (1994), a pesquisa em questão pode ser classificada como exploratória. Essa classificação justifica-se, pois o trabalho visa proporcionar maior compreensão do problema proposto (desenvolvimento dos municípios mineiros). Em relação às técnicas estatísticas, são utilizadas a análise de cluster e a análise de regressão por Mínimos Quadrados Ordinários (MQO). É utilizado o método two-step cluster com auxílio do software estatístico SPSS (Statistical Package for the Social Sciences) versão 21. O modelo de regressão é analisado com a utilização do software estatístico Eviews (Econometric Views) versão 5.0 .

Quanto aos dados, foi utilizado o universo de municípios de pequeno porte do estado de Minas Gerais. Nesta pesquisa, o porte do município foi definido com base no tamanho de sua população. Diante disso, foram classificados como pequenos municípios aqueles com população de até 50.000 habitantes no ano de 2010, de acordo com os dados do Censo Demográfico desse mesmo ano. Dos 853 municípios, 787 atendiam a esse critério. $\mathrm{Na}$ análise de cluster, são analisados os dados de 2010 para esses municípios e na análise de regressão são avaliadas as for- 
mações dos anos de 2000 a 2010 para o mesmo grupo de municípios.

\section{Análise de cluster}

A análise de cluster ou de conglomerados é uma técnica de análise exploratória destinada a revelar agrupamentos naturais em dado conjunto de informações. Para Corrar, Paulo e Dias Filho (2012), a opção pela análise de cluster justifica-se pela possibilidade de agrupar um conjunto de municípios a partir de suas similaridades, que podem ser explicadas pela proximidade entre os perfis dos municípios analisados em termos das variáveis utilizadas. Ressalta-se que o resultado obtido é completamente dependente das variáveis usadas como base de medida da similaridade.

Explica-se a escolha pelo procedimento two-step cluster porque o algoritmo empregado por esse procedimento tem várias características desejáveis, que o diferencia de técnicas de agrupamento tradicionais, como a consideração de que as variáveis são não correlacionadas e admitem uma distribuição normal, permitindo a análise tanto de variáveis categóricas quanto contínuas. As variáveis não necessitam ser previamente padronizadas como em outros métodos de análise. O sistema avalia as variáveis incluídas e padroniza as que forem necessárias antes da definição dos agrupamentos. Além disso, esse procedimento seleciona de forma automática o número de clusters com base em critérios estatísticos e permite analisar um número grande de dados.

No caso do software SPSS, versão 21 , a medida de avaliação do cluster é dada pela medida de silhueta de coesão e separação.
O coeficiente da silhueta é uma média de todos os casos individuais analisados a partir da relação da variação entre os clusters e em cada cluster. A medida da silhueta é um coeficiente que varia de -1 , indicando um modelo muito pobre, e 1 , indicando um excelente modelo. Um bom modelo de agrupamento tem uma silhueta média igual e superior a 0,5 . Valores abaixo de 0,5 indicam que o agrupamento não é muito bom. Valores inferiores a 0,2 mostram que os dados não exibem estrutura de agrupamento. Outro recurso disponível são os campos de avaliação. Por esse recurso, é possível obter informações sobre os clusters a partir de variáveis que não foram utilizadas na sua criação (IBM, n.d.).

A seleção das variáveis foi feita com base em aspectos teóricos da abordagem do desenvolvimento humano e conceitos relativos a fatores favoráveis à promoção do bem-estar da população, condição econômica e capacidade fiscal do município. As variáveis escolhidas para a definição dos agrupamentos são: Índice de Desenvolvimento Humano, Produto Interno Bruto per capita, Índice de Gini, Receita líquida per capita e Percentual da população em domicílio com banheiro e água encanada. Para compor o campo de avaliação foram escolhidas as variáveis: renda per capita, o percentual de pessoas de 18 anos ou mais com ensino fundamental completo, a esperança de vida, razão de dependência, taxa de desocupação 10 anos ou mais e valor adicionado na indústria, serviços e agropecuária. As siglas utilizadas para cada variável e as respectivas fontes das informações estão descritas no quadro 1. 
Quadro 1. Variáveis analisadas no modelo de cluster

\begin{tabular}{|c|c|c|c|}
\hline Função & Sigla & Descrição & Fonte \\
\hline \multirow{5}{*}{$\begin{array}{l}\text { Definição do } \\
\text { Cluster }\end{array}$} & IDHM & $\begin{array}{l}\text { Índice de Desenvolvimento } \\
\text { Humano Municipal }\end{array}$ & $\begin{array}{l}\text { Atlas do Desenvolvimento Hu- } \\
\text { mano (2013) }\end{array}$ \\
\hline & PIB pc & Produto Interno Bruto per capita & IMRS (2013) \\
\hline & GINI & Índice de Gini & $\begin{array}{l}\text { Altas do Desenvolvimento Hu- } \\
\text { mano (2013) }\end{array}$ \\
\hline & RECLIQ & Receita líquida per capita & IMRS (2013) \\
\hline & BAN_AGUA & $\begin{array}{l}\text { Percentual da população que vive } \\
\text { em domicílios com banheiro e água } \\
\text { encanada }\end{array}$ & $\begin{array}{l}\text { Altas do Desenvolvimento Hu- } \\
\text { mano (2013) }\end{array}$ \\
\hline \multirow{8}{*}{$\begin{array}{l}\text { Campos de } \\
\text { avaliação }\end{array}$} & RENDA PC & Renda per capita & $\begin{array}{l}\text { Altas do Desenvolvimento Hu- } \\
\text { mano (2013) }\end{array}$ \\
\hline & FUND COMP & $\begin{array}{l}\text { Percentual de pessoas de } 18 \text { anos } \\
\text { ou mais com fundamental completo }\end{array}$ & $\begin{array}{l}\text { Altas do Desenvolvimento Hu- } \\
\text { mano (2013) }\end{array}$ \\
\hline & ESP VIDA & Esperança de vida ao nascer & $\begin{array}{l}\text { Altas do Desenvolvimento Hu- } \\
\text { mano (2013) }\end{array}$ \\
\hline & RAZ DEP & Razão de Dependência & $\begin{array}{l}\text { Altas do Desenvolvimento Hu- } \\
\text { mano (2013) }\end{array}$ \\
\hline & TXDESOC & Taxa de desocupação & IMRS (2013) \\
\hline & VA_IND & $\begin{array}{l}\text { Participação percentual da indústria } \\
\text { no valor adicionado }\end{array}$ & IMRS (2013) \\
\hline & VA_SERV & $\begin{array}{l}\text { Participação percentual dos servi- } \\
\text { ços no valor adicionado }\end{array}$ & IMRS (2013) \\
\hline & VA_AGR & $\begin{array}{l}\text { Participação percentual da agrope- } \\
\text { cuária no valor adicionado }\end{array}$ & IMRS (2013) \\
\hline
\end{tabular}

O IDHM e o Índice de Gini foram escolhidos, respectivamente, como proxy para o desenvolvimento humano e grau de concentração de renda. O PIB per capita foi escolhido como proxy da condição econômica do município e o percentual de população com banheiro e água encanada como proxy de infraestrutura social. Os campos de avaliação, renda per capita, percentual de pessoas de 18 anos ou mais com fundamental completo e esperança de vida são medidas, respectivamente, das capacidades de poder aquisitivo, de educação e longevidade que indicam o contexto do desenvolvimento humano. O campo de avaliação razão de dependência traz informações importantes sobre o perfil populacional que influência a dinâmica econômica. A participação no valor adicionado da indústria, dos serviços e da agropecuária mostra o perfil da atividade econômica do município.

A proposta de desenvolvimento humano local tem como alvo o processo de ampliação das escolhas e liberdades das pessoas para que elas tenham capacidades e oportunidades 
para ser aquilo que desejam ser em cada município (variável proxy IDHM). Também é importante o papel da gestão pública municipal, responsável pela provisão de serviços, relacionados à capacidade de levar uma vida longa e saudável (variáveis proxys ESP VIDA e BAN_AGUA) e ter acesso a conhecimento (variável proxy FUND COMP). A condição de resposta do município refere-se à sua capacidade fiscal (variável proxy RECLIQ) e de gestão (BAN-AGUA). Volta-se à importância do crescimento econômico, pois a disponibilidade de recursos do município depende da arrecadação, que está relacionada ao dinamismo econômico (variáveis proxys PIBpc, VA_IND, VA_SERV, VA_AGR, RAZDEP), da mesma forma que a oportunidade do indivíduo de desfrutar de um padrão de vida digno está ligado à sua renda (variáveis proxys RENDA PC, GINI, TX_DES).

Diante disso, propõe-se uma tipologia para os municípios em termos de Desenvolvimento Humano Local, a partir da avaliação da condição social, econômica e fiscal do município, como mostra o quadro 2.

Quadro 2. Tipologia de classificação dos municípios

\begin{tabular}{|c|c|c|c}
\hline \multirow{2}{*}{$\begin{array}{c}\text { Características do } \\
\text { município }\end{array}$} & $\begin{array}{c}\text { Desenvolvimento } \\
\text { humano local baixo }\end{array}$ & $\begin{array}{c}\text { Desenvolvimento } \\
\text { humano local } \\
\text { intermediário }\end{array}$ & $\begin{array}{c}\text { Desenvolvimento } \\
\text { humano local } \\
\text { alto }\end{array}$ \\
\hline $\begin{array}{c}\text { Condição social } \\
\begin{array}{c}\text { Condição } \\
\text { econômica } \\
\text { Capacidade fiscal }\end{array}\end{array}$ & BAIXA & INTERMEDIÁRIA & ALTA \\
\hline
\end{tabular}

A proposta é avaliar cada um dos indicadores de forma relativa. Assim, a posição baixa, alta ou intermediária referem-se aos valores médios de cada variável analisada em cada cluster. Caso haja mais de três clusters, buscam-se subclassificações dentro das três classificações definidas.

\section{Análise de regressão}

A análise de regressão consiste em definir uma função matemática que busca descrever o comportamento de determinada variável, denominada dependente, com base nos valores de uma ou mais variáveis, classificadas como independentes (Corrar,
Paulo \& Dias Filho, 2012). Entre os modelos regressão possíveis, será utilizado neste estudo o modelo linear.

Segundo Fávero, Belfiore, Silva \& Chan (2009), a regressão linear tem como propósito analisar a relação entre duas ou mais variáveis explicativas, que se apresentam na forma linear, e uma variável dependente. O estudo da dependência de uma variável em relação a mais de uma variável explicativa (preditora) é conhecido como análise de regressão múltipla.

Tem-se, assim, uma especificação matemática para a relação de dependência de uma 
variável $(Y)$ quanto às variáveis explicativas $(X)$ definidas como função de regressão populacional (FRP):

\section{Equação 1}

$Y_{i}=\beta_{1}+\beta_{2} X_{i}+u_{i}$

Nessa equação, $\beta_{1} e \beta_{2}$ são os parâmetros da regressão e $u_{i}$ é o erro da regressão. A equação mostra que $Y_{i}$ depende tanto de $X_{i}$ quanto de $u_{i}$, por isso o modo de especificação desses termos é importante para a realização de qualquer inferência estatística sobre a variável dependente e sobre os parâmetros. Além disso, os verdadeiros valores de $\beta_{1} e \beta_{2}$ são na maioria das vezes desconhecidos, pois o que se tem nas avaliações práticas são amostras de valor $Y$ correspondentes a alguns valores $X \mathrm{~s}$ fixos. Nesse caso, a análise de regressão busca fazer inferências sobre os verdadeiros parâmetros populacionais (Gurajati, 2000).

Para analisar a dinâmica da variação do desenvolvimento humano dos pequenos municípios, em Minas Gerais entre 2000 e 2010, medido pelo IDHM, esta pesquisa adotou o modelo de regressão multivariada populacional, com base no Método dos Mínimos Quadrados Ordinários (MQO), expresso na equação 2.
Sendo que:

IDHM i 2000 e IDHM i 2010 são os índices de desenvolvimento humano do município i, observados, respectivamente, para os anos entre 2000 e 2010;

PIBpci00 é o Produto Interno Bruto (PIB) per capita do município i no ano de 2000 (valores correntes);

GINli00 é a variável Índice de Gini para o município i em 2000;

RECLIQi00 é a Receita Líquida per capita do município i em 2000 (valores correntes);

POP2000i00 é a variável população total para o município i em 2000;

$\beta_{0} \ldots \beta_{5}$ são os parâmetros estimados pelo modelo de regressão

$u_{i}$ indica o erro da regressão, com base nas pressuposições usuais.

A variável dependente corresponde à variação logarítmica do índice de desenvolvimento humano municipal entre os anos $2000 \mathrm{e}$ 2010.

No caso do parâmetro $\beta_{1}$, estimado a partir do logaritmo do índice de desenvolvimento humano municipal (IDHM) em 2000, indica que, por exemplo, se o parâmetro estimado for negativo, , há uma tendência de convergência entre os índices municipais de desenvolvimento humano, ao longo do período (2000-2010).

\section{Equação 2}

$$
\begin{aligned}
& \ln \left(\frac{I D H M_{i 2010}}{I D H M_{i 2000}}\right)=\beta_{0}+\beta_{1} \cdot \ln \left(I D H M_{i 00}\right)+\beta_{2} \cdot \ln \left(P I B p c_{i 00}\right)+\beta_{3} \cdot G I N I_{i 00}+ \\
& \beta_{4} \cdot R \text { RCLIQ } \\
& i 00
\end{aligned}
$$




\section{DESENVOLVIMENTO HUMANO LOCAL: UMA ANÁLISE MULTIVARIADA PARA OS PEQUENOS MUNICÍPIOS EM MINAS GERAIS}

Para o parâmetro $\beta_{2}$, há expectativas de que seu sinal seja positivo com base na discussão teórica, refletindo uma relação direta entre a variação do IDHM e o valor PIB per capita entre os diversos municípios em 2000. O PIB per capita é uma variável proxy da condição econômica, fator importante para a promoção do desenvolvimento humano. Caso essa relação seja verdadeira, é possível afirmar que o IDHM tem crescido mais intensamente nos municípios que apresentam historicamente os maiores valores de PIB per capita (vice-versa). Isso implicaria reconhecimento de que os municípios mais pobres economicamente teriam maiores dificuldades para melhorar seu desempenho em termos do desenvolvimento humano. Essa dificuldade poderia implicar que uma região mais pobre teria um ritmo mais lento em comparação a uma região rica em relação ao aumento do IDHM.

Sobre o parâmetro $\beta_{3}$, espera-se que seu sinal seja negativo, refletindo uma relação inversa entre as taxas de crescimento do IDHM e o valor do Índice de Gini (proxy para o nível de desigualdade de renda). A equidade aparece como um fator importante para o desenvolvimento humano. Quanto mais bem distribuída a renda, maiores as chances do crescimento se traduzir em bem-estar (PNUD,1996). Caso essa correlação se revele, é possível afirmar que o IDHM tem crescido com maior intensidade nos municípios que apresentam historicamente os menores valores de GINI (vice-versa). Isso implicaria o reconhecimento de que os municípios mais desiguais em termos da renda (maior GINI) teriam maiores dificuldades para melhorar seu desempenho relativo em termos do desenvolvimento humano.
A respeito do parâmetro $\beta_{4}$, há perspectivas de que seu sinal seja positivo, refletindo uma relação direta entre as taxas de crescimento do IDHM e o valor da receita líquida per capita (valor da receita orçamentária, deduzidas as operações de crédito, da administração municipal, dividido pela população total) no ano 2000. A receita líquida per capita é uma medida potencial da capacidade de o município executar serviços essenciais à população. Verificando-se essa relação, é possível afirmar que o IDHM tem crescido mais intensamente nos municípios que apresentam historicamente os maiores valores de receita líquida per capita (vice-versa). Isso implicaria o reconhecimento de que os municípios com recursos fiscais mais escassos teriam maiores dificuldades para melhorar seu desempenho em termos do desenvolvimento humano.

A variável POP objetiva controlar os efeitos das variáveis IDHM, PIBpc, GINI, RECLIQ sobre a variável de resposta, evitando-se a ocorrência de regressão espúria. As variáveis $\frac{I D H M_{2010}}{I D H M_{2000}}$,IDHM, PIBPc, RECLIQ e POP foram consideradas em seus logaritmos naturais (In), de maneira a permitir melhor ajustamento da regressão e facilitar a interpretação de seus parâmetros.

Em razão da presença de heteroscedasticidade, muitas vezes comum em dados de seção cruzada e de grande variabilidade dos dados, a significância dos parâmetros foi testada pelo método de correção de White. 


\section{ANÁLISE DOS RESULTADOS}

\section{Minas Gerais}

Minas Gerais é um estado muito heterogêneo, com 19.597.330 habitantes, segundo os dados do IBGE de 2010. A renda e a população estão distribuídas em 853 municípios (estado com o maior número de municípios do país), com distintos perfis. Alguns de considerável dinamismo econômico e elevados indicadores sociais e outros pouco desenvolvidos, estagnados e com péssimos indicadores sociais.

Os valores agregados mostram que $92 \%$ dos municípios têm até 50.000 habitantes e concentram $43 \%$ da população e $30 \%$ do PIB. Desagregando essa informação por faixas populacionais, em $2010,28,14 \%$ dos municípios tinham até 5.000 habitantes; $29,43 \%$ de 5.001 a $10.000 ; 21,57 \%$ de 10.001 a 20.000 e $13,13 \%$ de 20.001 a 50.000 habitantes. Considerando os municípios com até 20.000 , tem-se quase $80 \%$ dos municípios e
$26,25 \%$ da população, ou seja, um quarto da população mineira concentra-se em municípios com até 20.000 habitantes. Já os municípios entre 50.001 e 100.000 são $4 \%$ do total de municípios e detêm $13,5 \%$ da população e $14,5 \%$ do PIB. Já os municípios com mais de 100.000 habitantes representam 3\% e agregam $43 \%$ da população e $55 \%$ do PIB. Só a capital Belo Horizonte congrega $12 \%$ da população e $15 \%$ do PIB.

\section{Análise de cluster}

Com base nas variáveis IDHM, PIB pc, GINI, RECLIQ e BAN_AGUA e no método two-step cluster, foram definidos três clusters seguindo o critério do melhor ajuste de coesão e separação (silhueta de 0,5 ).

O primeiro agrupamento é formado por $25,2 \%$ dos municípios (198), o agrupamento 2 por $71,2 \%$ dos municípios (564) e o agrupamento 3 por 3,2\% (25) municípios. Os resultados para os clusters formados podem ser vistos na tabela 1. 
Tabela 1. Resultados dos clusters, pequenos municípios - Minas Gerais - 2010

\begin{tabular}{|c|c|c|c|c|c|}
\hline \multirow{3}{*}{$\begin{array}{c}\text { Características do } \\
\text { município }\end{array}$} & \multirow{3}{*}{\multicolumn{2}{|c|}{ Variáveis }} & \multicolumn{3}{|c|}{ Cluster } \\
\hline & & & 1 & 2 & 3 \\
\hline & & & $\begin{array}{c}\text { Desenvolvimento } \\
\text { humano local } \\
\text { baixo }\end{array}$ & $\begin{array}{l}\text { Desenvolvimento } \\
\text { humano local } \\
\text { intermediário }\end{array}$ & $\begin{array}{l}\text { Desenvolvimento } \\
\text { humano local alto }\end{array}$ \\
\hline \multirow{12}{*}{ Condição social } & \multirow{2}{*}{ IDHM } & Média & 0,61 & 0,68 & 0,70 \\
\hline & & Desvio padrão & 0,03 & 0,03 & 0,03 \\
\hline & \multirow{2}{*}{ RENDA_PC } & Média & 306,80 & 518,44 & 615,10 \\
\hline & & Desvio padrão & 58,49 & 126,88 & 150,19 \\
\hline & \multirow{2}{*}{ FUND_COMP } & Média & 29,90 & 38,37 & 43,98 \\
\hline & & Desvio padrão & 5,10 & 6,65 & 7,81 \\
\hline & \multirow{2}{*}{ ESP_VIDA } & Média & 72,60 & 74,81 & 75,61 \\
\hline & & Desvio padrão & 1,32 & 1,50 & 1,44 \\
\hline & \multirow{2}{*}{ GINI } & Média & 0,50 & 0,46 & 0,45 \\
\hline & & Desvio padrão & 0,04 & 0,05 & 0,07 \\
\hline & \multirow{2}{*}{ BAN_AGUA } & Média & 75,73 & 96,78 & 97,53 \\
\hline & & Desvio padrão & 11,75 & 3,82 & 3,19 \\
\hline \multirow{12}{*}{$\begin{array}{l}\text { Condição } \\
\text { econômica }\end{array}$} & \multirow{2}{*}{ PIBpc } & Média & 6036,83 & 11510,21 & 57092,70 \\
\hline & & Desvio padrão & 2292,00 & 6546,58 & 53236,53 \\
\hline & \multirow{2}{*}{ VA_INDP } & Média & 11,78 & 16,62 & 47,43 \\
\hline & & Desvio padrão & 5,34 & 13,13 & 32,33 \\
\hline & \multirow{2}{*}{ VA_SERVP } & Média & 65,04 & 56,13 & 36,32 \\
\hline & & Desvio padrão & 9,76 & 12,81 & 22,57 \\
\hline & \multirow{2}{*}{ VA_AGR } & Média & 23,18 & 27,25 & 16,25 \\
\hline & & Desvio padrão & 10,47 & 14,97 & 18,67 \\
\hline & \multirow{2}{*}{ RAZDEP } & Média & 57,09 & 47,26 & 44,24 \\
\hline & & Desvio padrão & 5,71 & 4,05 & 3,87 \\
\hline & \multirow{2}{*}{ TX_DESO } & Média & 7,87 & 5,89 & 5,75 \\
\hline & & Desvio padrão & 3,26 & 3,04 & 2,43 \\
\hline \multirow[b]{2}{*}{ Capacidade Fiscal } & \multirow[b]{2}{*}{ RECLIQ } & Média & 1311,67 & 1513,55 & 3854,34 \\
\hline & & Desvio padrão & 338,62 & 547,14 & 1923,40 \\
\hline
\end{tabular}

Fonte: Resultados da pesquisa.

Cluster 1: Desenvolvimento humano local baixo

É formado pelos municípios de piores condições nas três dimensões avaliadas. Na condição social, o IDHM médio apresenta valor de 0,61. Desmembrando as informações contidas no IDHM, percebe-se uma grande diferença em termos da renda per capita. Em média, um indivíduo desse cluster ganha metade da renda per capita média ( $R \$$ 306,80 ) de um indivíduo do agrupamento 3 ( $R \$ 615,10)$. A situação educacional, avaliada com base no percentual de pessoas com
18 anos ou mais com ensino fundamental completo, também é bem discrepante em relação aos demais agrupamentos: menos de $30 \%$ das pessoas com mais de 18 anos tem ensino fundamental completo. A esperança de vida ao nascer para uma pessoa desse cluster $(72,60)$ é em média três anos a menos de um indivíduo pertencente ao cluster 3 . A concentração de renda, medida pelo GINI médio, também é a maior dos três grupos $(0,50)$. O acesso a domicílio com banheiro e água encanada é, em média, usufruído por apenas $75 \%$ das pessoas nos municípios. No aspecto econômico, o PIB é 
em média $R \$ 6.036,83$, pouco mais da metade do valor do PIB médio do agrupamento 2. Na composição das atividades econômicas há, em média, baixa participação da indústria no valor adicionado, $11,78 \%$. O valor adicionado dos serviços é $65 \%$. Nessa medida, estão incluídos os serviços públicos, que, em municípios com baixo dinamismo econômico, são grandes responsáveis pela geração de empregos. A participação da agropecuária no valor adicionado é $23,18 \%$. A razão de dependência é $57 \%$, o que revela uma situação crítica em termos econômicos, pois há um grande número de dependentes. A taxa de desocupação apresenta a maior média do grupo, 7,87\%. A capacidade fiscal do município, medida em termos da receita líquida per capita $(\mathrm{R} \$ 1.311,67)$, é a menor entre os três agrupamentos.

\section{Cluster 2: Desenvolvimento humano local intermediário}

É formado pelos municípios de condições intermediárias nas três dimensões avaliadas. $\mathrm{Na}$ condição social, o IDHM apresenta valor de 0,68 , valor médio bem acima do cluster $1(0,61)$ e bem próximo do cluster $3(0,70)$. Avaliando seus componentes, apreende-se que há uma diferença em termos da renda per capita. Em média, um indivíduo desse agrupamento ganha $84 \%$ da renda per capita média de um indivíduo do agrupamento 3. A avaliação do percentual de pessoas com 18 anos ou mais com ensino fundamental mostra que $38 \%$ das pessoas com mais de 18 anos tem ensino fundamental completo, um valor percentual seis pontos abaixo do agrupamento 3. A esperança de vida ao nascer para uma pessoa desse cluster é, em média, de 74,8 anos. A concentração de renda, medida pelo valor médio do GINI,
0,46 é bem próxima do valor médio do GINI do grupo $3(0,45)$. O acesso a domicílios com banheiro e água encanada é, em média, usufruído por quase $97 \%$ das pessoas nos municípios que compõem esse agrupamento. No aspecto econômico, o PIB é em média de $R$ \$ 11.510,21, valor bem abaixo do valor do PIB médio do agrupamento $3(\mathrm{R} \$ 57.092,70)$. $\mathrm{Na}$ composição das atividades econômicas há, em média, baixa participação da indústria no valor adicionado - 16,62\% -, elevada participação dos serviços - $56,13 \%$ - e uma participação da agropecuária superior aos demais grupos - $27,25 \%$. A razão de dependência é $47 \%$, o que revela uma situação em que há um maior número de pessoas economicamente ativas em comparação ao número de pessoas dependentes. Por fim, a taxa de desocupação - 5,89\% -, está bem próxima da apresentada pelo agrupamento 3 . A capacidade fiscal do município, medida em termos da receita líquida per capita - $\mathrm{R} \$ 1.513,55-$, é $15 \%$ superior ao valor do agrupamento 1 , mas muito inferior ao valor apresentado pelo agrupamento $3(R \$ 3.834,54)$, pouco mais de um terço.

Cluster 3: Desenvolvimento humano local alto

É formado pelos municípios de elevadas condições nas três dimensões avaliadas. $\mathrm{Na}$ condição social, o IDHM apresenta valor de 0,70 , valor médio ligeiramente superior ao cluster $2(0,68)$ e bem alto em relação ao cluster $1(0,61)$. Avaliando seus componentes, verifica-se que há uma diferença em termos da renda per capita. Um indivíduo desse agrupamento ganha $R \$ 615,10$, o dobro, em termos da renda per capita média de um indivíduo do agrupamento 1 ( $R \$ 306,80)$. A avaliação da educação dos adultos mostra 
que $44 \%$ das pessoas com 18 anos ou mais têm ensino fundamental completo. Esse resultado é o melhor entre os três grupos analisados. A esperança de vida ao nascer para uma pessoa desse cluster é 75,6 anos. A concentração de renda, medida pelo GINI, é 0,45 . O percentual de domicílio com banheiro e água encanada é, em média, de $98 \%$. No aspecto econômico, o PIB per capita é em média de $R \$ 57.092,70$, valor quase cinco vezes maior que o PIB per capita médio do agrupamento 2 e quase dez vezes maior que o PIB médio do agrupamento 1. $\mathrm{Na}$ composição das atividades econômicas há, em média, grande participação da indústria no valor adicionado $(47,43 \%)$. A participação dos serviços no valor adiciona- do é $36,32 \%$ e, na agropecuária, $16,25 \%$. A razão de dependência é, em média, $44 \%$ e, a taxa de desocupação, $5,75 \%$, ambos os menores percentuais em comparação aos clusters 1 e 2. A capacidade fiscal do município, medida em termos da receita líquida per capita - $R \$ 3.854,345$ - é a maior entre os demais agrupamentos, quase três vezes maior.

Um aspecto interessante desses clusters é a localização dos municípios que os compõe, que ultrapassa a divisão em mesorregiões do IBGE. Percebe que há regiões distantes geograficamente e próximas que se assemelham em termos dos atributos definidos como mostra a figura 1.

Figura 1. Cluster dos pequenos municípios em Minas Gerais - 2010

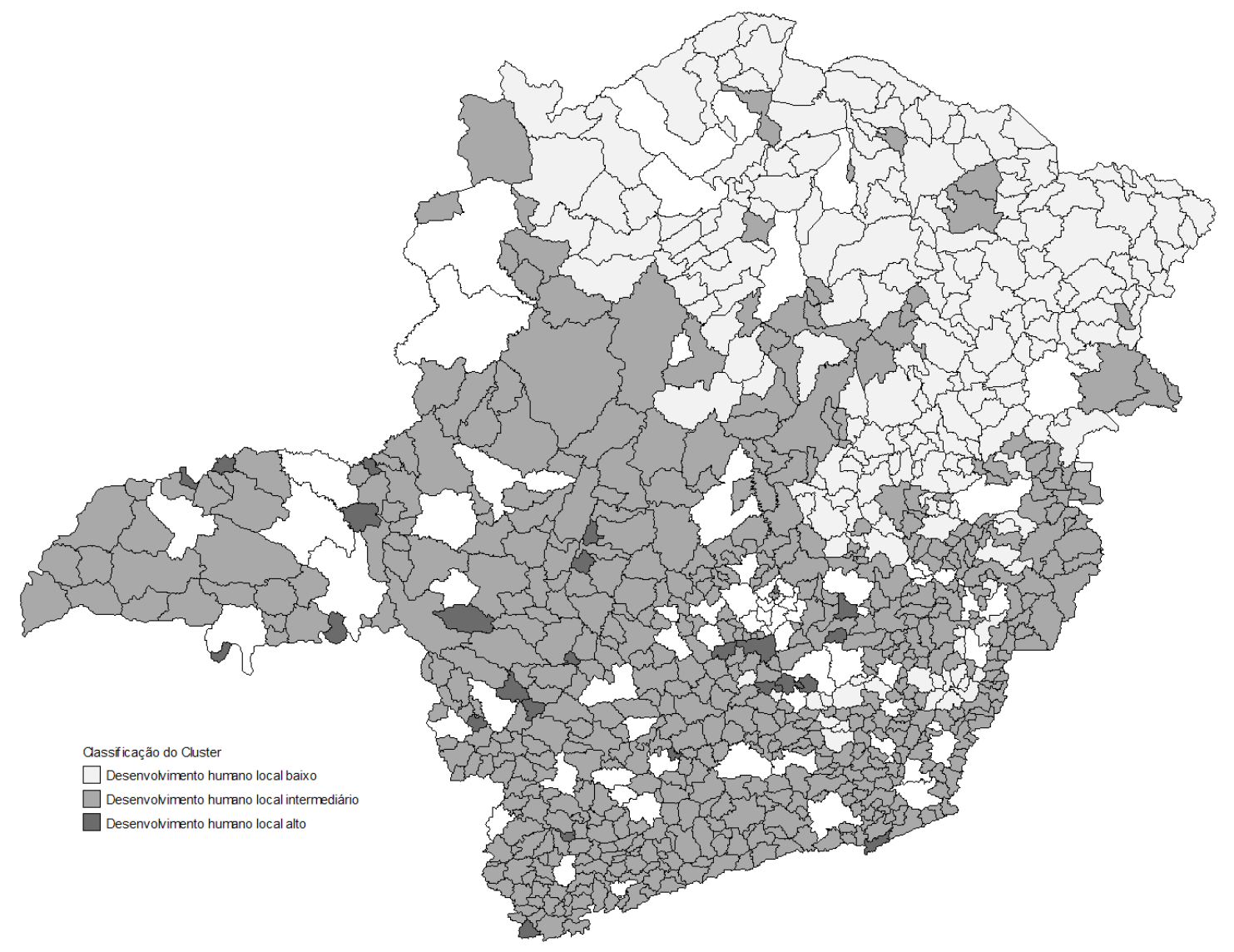


Análise de regressão

A tabela 2 mostra os resultados do modelo de regressão utilizado para analisar a dinâ-

Tabela 2. Resultados das estimativas mica da variação relativa do índice de desenvolvimento humano entre os pequenos municípios em Minas Gerais.

\begin{tabular}{|c|c|c|c|c|}
\hline Variável & Coeficiente & Erro padrão* & Estatística "t" & Probabilidade \\
\hline Intercepto & $\beta_{0}=-0.38929$ & 0.041949 & -9.280 .142 & 0.0000 \\
\hline $\operatorname{Ln}\left(I D H M_{i o o}\right)$ & $\beta_{1}=-0.56671$ & 0.013025 & -4.350 .935 & 0.0000 \\
\hline $\operatorname{Ln}(P I B p c$ ioo $)$ & $\beta_{2}=0.00944$ & 0.003229 & 2.923 .332 & 0.0036 \\
\hline Gini & $\beta 3=-0.09829$ & 0.016949 & -5.799 .314 & 0.0000 \\
\hline $\operatorname{Ln}\left(R E C L I Q_{i 00}\right)$ & $\beta 4=0.01606$ & 0.004673 & 3.436 .303 & 0.0006 \\
\hline $\operatorname{Ln}\left(P O P{ }_{i 00}\right)$ & $\beta 5=0.01372$ & 0.002247 & 6.108 .285 & 0.0000 \\
\hline \multirow[t]{4}{*}{$N=787$} & & \multicolumn{2}{|l|}{$\mathrm{R}^{2}$} & 0.844985 \\
\hline & & \multicolumn{2}{|l|}{$\mathrm{R}^{2}$ ajustado } & 0.843993 \\
\hline & & \multicolumn{2}{|l|}{ Estatística "F" } & 8.514 .468 \\
\hline & & \multicolumn{2}{|c|}{ Probabilidade(Estatística "F") } & 0.000000 \\
\hline
\end{tabular}

De forma geral, como mostra a tabela, os resultados confirmam a validade estatística do modelo de regressão proposto. $O$ teste "F" foi significativo a $1 \%$, o que indica a significância dos valores dos coeficientes de ajustamento da regressão, R2 e R2 ajustados. O coeficiente de ajustamento R2 indica que pelo menos $84,5 \%$ das variações relativas do índice de desenvolvimento humano estão correlacionadas às variáveis independentes do modelo. Os testes " $\mathrm{t}$ " foram significativos a $1 \%$ para todos os parâmetros.

O parâmetro estimado $\beta_{1}$ apresentou sinal negativo, o que mostra que os municípios com menor índice de desenvolvimento humano são os que têm apresentado as maiores taxas de crescimento relativo do IDH entre 2000 e 2010 (e vice e versa). Desse modo, se esse movimento percebido para o período de 2000 a 2010 continuar, há uma tendência de redução das desigualdades em termos do IDHM entre os pequenos municípios em Minas Gerais.

Já o parametro estimado $\beta_{2}$ também se mostrou com sinal positivo, o que indica que a variação relativa nas taxas de crescimento do índice de desenvolvimento humano são maiores naqueles municípios que apresentavam maiores PIB per capitas, em 2000, ou seja, melhor condição econômica. Essa constatação reforça o argumento da importância da condição econômica do município para promoção do desenvolvimento econômico como apontado nas discussões feitas pelo PNUD (1996). Por outro lado, o sinal positivo do parâmetro $\beta_{2}$ evidencia que as maiores taxas de crescimento no índice de desenvolvimento humano ocorreram nos municípios historicamente mais avançados economicamente (e vice-versa), o que reforça as desigualdades. Assim, controlando pelas demais variáveis preditoras, a variação 


\section{DESENVOLVIMENTO HUMANO LOCAL: UMA ANÁLISE MULTIVARIADA PARA OS PEQUENOS MUNICÍPIOS EM MINAS GERAIS}

de $1 \%$ no PIB per capita pode elevar a variação do IDHM em 0,09\%.

O parâmetro estimado $\beta_{3}$, que apresentou sinal negativo, mostra que as taxas de crescimento do índice de desenvolvimento humano são maiores naqueles municípios que apresentavam menores valores no índice de Gini, em 2000, ou seja, com menor concentração de renda. Essa constatação confirma a proposição teórica de que a menor desigualdade de renda favorece a incorporação de um maior número de pessoas aos benefícios advindos da melhoria econômica, que se revelam nos melhores indicadores de renda, saúde e educação (componentes do IDHM). Dessa forma, em uma perspectiva preditiva, controlando pelas demais variaveis preditoras, a redução de um ponto no GINI eleva a variação do IDHM em 0,09\%.

O parâmetro estimado $\beta_{4}$ mostrou-se com sinal positivo, o que indica que as taxas de crescimento do índice de desenvolvimento humano são maiores naqueles municípios que apresentavam maiores valores de receita líquida per capita, em 2000. Espera-se, assim, que municípios com maior receita líquida per capita (receitas próprias e transferências intergovernamentais) tenham melhores resultados em termos de desenvolvimento humano. Nessa perspectiva, controlando pelas demais variaveis preditoras, o aumento de $1 \%$ na receita líquida do município elevaria em $0,16 \%$ a variação do IDHM.

O parâmetro estimado $\beta_{5}$ indica que a variação nas taxas de crescimento do índice de desenvolvimento humano são maiores nos municípios que inicialmente apresentavam maior população $\left(\beta_{5}>0\right)$. Dessa forma, os municípios maiores teriam uma vantagem relativa aos demais municípios analisados em termos de crescimento do desenvolvimento humano.

Afere-se, portanto, que, para os municípios analisados, o PIB per capita, o Índice de Gini, a Receita líquida per capita e o tamanho populacional se apresentaram como variáveis explicativas significativas para 0 comportamento da variação do IDHM.

\section{CONSIDERAÇÕES FINAIS}

O objetivo deste estudo foi explorar de forma comparativa e classificatória a realidade socioeconômica dos pequenos municípios em Minas Gerais, sob a perspectiva da abordagem do desenvolvimento humano local, tomando como base as informações dos anos de 2000 a 2010.

O ponto de partida foi a compreensão da abordagem do desenvolvimento humano, sua contextualização histórica e sua aplicação no âmbito local. Essa proposta é importante, pois, apesar da grande gama de possibilidades de intervenção em âmbito nacional, o espaço local (município) assume papel relevante.

Nessa discussão, destaca-se o papel da gestão pública municipal, principalmente após a constituição de 1988, quando o município assume a responsabilidade de provisão de serviços de saúde e educação estritamente relacionados às capacidades de levar uma vida longa e saudável (longevidade) e ter acesso ao conhecimento (educação). A condição de resposta do município está ligada à sua condição financeira e de 
Valéria de Jesus Coelho Ferreira dos Santos - Frederico Poley Martins Ferreira

gestão.

Volta-se, assim, para a essencialidade do crescimento econômico, pois a disponibilidade de recursos do município depende da arrecadação, que está relacionada ao dinamismo econômico. Da mesma forma que a oportunidade do indivíduo de desfrutar de um padrão de vida digno vincula-se à sua renda.

A análise de cluster permitiu a classificação dos municípios a partir da sua condição de desenvolvimento humano local em três categorias de análise. A localização desses clusters extrapola a divisão em mesorregiões e revela pontos isolados de melhor condição socioeconômica em regiões tradicionalmente deprimidas, como o Vale do Jequitinhonha, Norte de Minas e Mucuri, municípios de baixo desenvolvimento humano em áreas com histórico de melhor desenvolvimento socioeconômico como a região Central Mineira e Metropolitana de Belo Horizonte. Essa constatação coloca em evidência um contexto de mudanças na dinâmica econômica do estado nas últimas décadas e a necessidade de avaliação dos fatores de avanço e retrocessos dos municípios envolvidos.

A análise de regressão, em uma perspectiva confirmatória da discussão teórica, mostrou que a taxa de crescimento do desenvolvimento humano, medido pela variação do IDHM, foi maior nos municípios com maior PIB per capita, menor valor no Índice de Gini, maior Receita líquida per capita e maior tamanho populacional.

Esse resultado reforça a importância já apontada pela literatura da condição econômica do município, em termos de PIB per capita, que se reflete na receita líquida per capita, uma vez que a arrecadação municipal é diretamente relacionada à atividade econômica do município. Assim, sendo os municípios economicamente deprimidos, eles se apresentam com maiores dificuldades para alcançar melhores condições de vida para sua população.

A questão do tamanho do município também é um ponto importante. A variação nas taxas de crescimento do índice de desenvolvimento humano são maiores nos municípios que inicialmente apresentavam maior população. Esse resultado expõe a fragilidade econômica e social dos pequenos municípios e traz para as discussões sobre a arrecadação fiscal, pacto federativo e políticas de desenvolvimento um elemento novo: a diversidade de contextos nos diferentes portes de municípios.

A partir do exposto, reforça-se a necessidade de se buscar um desenvolvimento equilibrado por meio do fortalecimento das ligações entre crescimento e desenvolvimento humano. Para tal, são essenciais políticas que favoreçam a expansão das capacidades dos indivíduos via ações efetivas de melhoria dos serviços de saúde e educação, que promovam a redução das iniquidades e das oportunidades de emprego e renda. Verificam-se também a importância dos pequenos municípios, sua viabilidade econômica e seus desafios, o que confere relevância às políticas públicas que fortaleçam a ação municipal.

\section{REFERÊNCIAS}

Abrucio, F., \& Couto, C. (1996). A redefinição do papel do estado no âmbito local. São Paulo em Perspectiva, 10(3), 40-47. Recuperado 


\section{DESENVOLVIMENTO HUMANO LOCAL: UMA ANÁLISE MULTIVARIADA PARA OS PEQUENOS MUNICÍPIOS EM MINAS GERAIS}

de http://produtos.seade.gov.br/produtos/ spp/v10n03/v10n03_05.pdf.

Boisier, S. (2001). Desarrollo (local): ¿De qué estamos hablando? In Madoery, O., \& Vázquez-Barquero, A. (Eds.), Transformaciones globales, Instituciones y Políticas de desarrollo local. Rosário: Homo Sapiens. Recuperado de http://abacoenred.com/IMG/ pdf/boisier.pd

Boueri, R, Monasterio, L. Mation, L., \& Silva, M. (2013). Multiplicai-vos e crescei? FPM, emancipação e crescimento econômico municipal. In Boueri, R. \& Costa, M. (Eds). Brasil em desenvolvimento 2013: Estado, planejamento e políticas públicas. Brasília: Ipea. Recuperado de http://www.ipea.gov.br/portal/index. php?option= com_content\&view= article\&id=20729

Buarque, S. (1999). Metodologia de planejamento do desenvolvimento local e municipal sustentável Material para orientação técnica e treinamento de multiplicadores e técnicos em planejamento local e municipal. Brasília, DF: Incra. Recuperado de http://www.iica.org.br/docs/publicacoes/publicacoesiica/ sergiobuarque.pdf

Cardoso, F. (1996). Que valores comandam hoje o processo de desenvolvimento? In PNUD. (1996). Relatório do Desenvolvimento Humano 1996. Lisboa, Portugal: Tricontinental.

Corrar, L., Paulo, E, \& Dias Filho, J. (Coord.). (2012). Análise multivariada para os cursos de Administração, Ciências Contábeis e Economia. São Paulo, SP: Atlas.

Dowbor, L. (2008). Políticas para o desen- volvimento local. São Paulo, SP: Perseu Abramo.

Fávero, L., Belfiore, P., Silva, F., \& Chan, B. (Coords). (2009). Análise de dados: modelagem multivariada para tomada de decisões. Rio de Janeiro, RJ: Elsevier.

Ferreira, F. (1996). As pequenas cidades e sua importância socioeconômica no Brasil atual. Revista Reuna 1(1), 41-45. Recuperado de http://revistas.una.br/index.php/reuna/ issue/view/45.

Fukuda-Parr, S. (2002). Operacionalizando as ideias de Amartya Sen sobre capacidades, desenvolvimento, liberdade e direitos humanos - o deslocamento do foco das políticas de abordagem do desenvolvimento humano. Recuperado de www.soo.sdr. sc.gov.br/index.php?option=com_contente\& task=view\&id=108\&ltemid=206.

Fundação João Pinheiro, Centro de Estatísticas e Informações. (2013). Índice Mineiro de Responsabilidade Social - IMRS 2013. Recuperado de www.fjp.mg.gov.br

Fundação João Pinheiro, Centro de Estatísticas e Informações. (2010). Produto Interno Bruto dos munic ípios de Minas Gerais 2010. Recuperado de www.fjp.mg.gov.br/index. php/docman/cei/pib/pib-municipais/164-informativo-pibmuni cipios-mg-2010/file

Gujarati, D. (2000). Econometria básica (3. ed). São Paulo, SP: Makron Books.

International Business Machines, Advanced Statistical Analys Using SPSS (n.d.). Recuperado de http://valeria-pc:62412/help/index.jsp?topic=/com.ibm.spss.statistics. help/ 
idh_twostep_main.htm

Programa das Nações Unidas para o Desenvolvimento, Fundação João Pinheiro, \& Instituto de Pesquisa Econômica Aplicada. (2013). Material de Apoio. In Atlas do Desenvolvimento Humano no Brasil 2013. Recuperado de www.pnud.org.br

Programa das Nações Unidas para o Desenvolvimento, Fundação João Pinheiro, \& Instituto de Pesquisa Econômica Aplicada. (1996). Relatório do Desenvolvimento Humano 1996. Lisboa, Portugal: Tricontinental.

Santos, F. (2013). A atuação dos governos locais na redução das desigualdades so- cioeconômicas. Tese de Doutorado, Escola de Administração de Empresas de São Paulo, São Paulo, São Paulo, Brasil. Recuperado de http://bibliotecadigital.fgv.br/dspace/ bitstream/handle/10438/10670/Tese\%20-\%2 Fernand \% 20 Burgos.pdf?sequence $=1$

Souza, C. (2002). Governos e sociedades locais em contextos de desigualdades e descentralização. Ciência \& Saúde Coletiva, 7(3), 431-441. doi:10.1590/S141381232002000300004

Veloso J., Monasterio L., Vieira R, \& Miranda R. (Org). (2011). Gestão municipal no Brasil: Um retrato das prefeituras. Brasília, DF: Ipea 